Japanese Psychological Research

1087 , Vil. 29. No. 3, 117-152

Short Report

\title{
The effect of information uncertainty on decision making
}

\author{
MAKI WADA ${ }^{1}$ \\ Department of Pspchology, College of Humanities and Sciences, Nihon Iniversily, Setagaya-ku, Tokvo 150
}

This study was conducted to examine (a) the effect of uncertainty of information on one's own ability (IS) and of the partner's ability (IGM) on task choice and choice sliti, and (b) the relationship of importance of each information to task choice and choice shift. Fifty-four undergraduates (26 males, 28 females) were first given IS (certain or uncertain) followed by the initial choice and then IGM (certain or uncertain) followed by the second choice. When IS and IGM were both certain or uncertain, no choice sift occurred, and importance of each information was seen nearly equal. Choice shift occurred when one information was certain and the other uncertain, its direction being a function of which information was certain. Importance of each information also differed between the combinations of uncertainty. It was suggested that uncertainty of cue information was a determinant of decision making and that the way to utilize it in decision making differed depending on its uncertainty.

Key words: uncertainty of cue information, imporatnce of cue information, task choice, choice shift, decision making.

The model of risk taking behavior proposed by Atkinson (1957) has been an important source of hypotheses about how people with different motives might behave in achievement situations where both success and failure are possible. In his model an affective value plays an important role in determination of task choice. Trope and Brickman (1975) and Trope and Ben-Yair (1982) maintained that the desire to assess one's own ability is a more powerful determinant of task choice. They suggested that people choose achievement tasks depending on the amount of information about their abilities gained from performance outcomes. This left the question of which value is the actual determinant of task choice: affective value or informational value? Raynor (1982) tried to extend and refine Atkinson's initial model and concluded that either affective or informational value can predominate depending on which aspects of the situations are emphasized by the researcher

1 The author wishes to thank Prof. Toshiro Suenaga (Nihon University) for his helpful suggestions on this paper. and which aspects are perceived more valuable by the individual who confronted with such a task. Therefore, if the subject's ability is estimated and the estimation is fed back to him or her, such feedback (Information of subject's own ability; IS) can be an important determinant of task choice in an achievement situation.

Now, how do cue information of different objects, information of one's own ability (IS) and that of other group member's ability (IGM) influence task choice? Information exchange among group members as a determinant of decision making was suggested as having an explanatory value for attitude changes (e.g. Anderson \& Graesser, 1976; Burnstine \& Vinokur, 1975; Goethals \& Zanna, 1979). The above studies suggested that the direction of consensus reached by individuals in a group is determined by contents, amounts, and characteristics of information. Kovacs (1982) manipulated uncertainty of information necessary for performing tasks and the pattern of information allocation within the group and found the latter affected the degree of choice shift. Therefore, uncertainty of IS and IGM and uncertainty 
combinations of them should determine task choice and direction of choice shift.

Thus, the present study was conducted to investigate how uncertainty of IS and IGM influences their utilization in task choice and what kind of task choice results from their utilization.

\section{Method}

\section{Subjects}

Fifty-four undergraduates (26 males, 28 females) of Nihon University were randomly assigned to one of four experimental conditions (certain vs. uncertain IS $\times$ certain vs. uncertain IGM).

\section{Task}

The anagram task designed by Tanaka (1970) was used as a choice task. It was to translate meaningless words of four syllables such as 'MEKUIWA' into meaningful words such as 'MEIWAKU' within given time limits.

\section{Procedure and Manipulations}

All 54 subjects participated together in the present experiment in a classroom setting and the experimenter was the author of this paper.

1. The ostensible explanations of the experiment given to the subjects was that it was designed to survey information integration ability by a "working test," which was in fact the anagram test of Tanaka (1970), and that this ability was divided into three levels: (a) lower, (b) intermediate, and (c) higher level. In order to minimize differences among subjects in initial beliefs about their ability, it was suggested that people could be classified into one of three ability levels regardless of age, sex, and career and that the ability could not be measured adequately by standard intelligence tests or achievement tests.

2. All subjects rated the chances of having each of the three ability levels on three 11 -point scales (ranging from $0 \%$ to $100 \%$ ). The sum of each probability estimation on the three scales was $100 \%$. These estimations were interpreted as the prior belief of their ability.

3. Subjects were shown the task, and informed that (a) the "working test" consisted of three groups of subordinate items in parallel with each ability level, and (b) the score was calculated by assigning 1,2 , and 3 to the items of lower, intermediate, and higher level. They then practiced a sample task for $3 \mathrm{~min}$. It consisted of 20 items and the subjects were fakely instructed that these items were almost equally divided into three items levels. After the practice they were left free to count how many items they had correctly resolved.

4. Subjects were given one of two slips presented in the form of the ability rating scales. One slip showed scales with $0 \%$ for lower, $80 \%$ for intermediate, and $20 \%$ for higher ability level, and the other with $20 \%$ for lower, $40 \%$ for intermediate, and $40 \%$ for higher ability level. The former was supposed to induce certain IS in the subjects and the latter to induce uncertain IS. The values on the scales were determined based on the results of the pilot study.

In the pilot study 32 male undergraduates rated the chances of having each of the three ability levels on the same three 11 -point scales as in the present study. Uncertainty of information was then indicated by computing the information theory indices. ${ }^{2}$ The probability distribution resulting in the minimum uncertainty value took $0 \%$ for lower, $80 \%$ for intermediate, and $20 \%$ for higher ability level, while for the maximum the distribution was $20 \%$, $40 \%$, and $40 \%$, respectively. The former

2 $U=-\sum P_{i} \log _{2} P_{i}$, where $U$ stands for uncertainty values and $P_{i}$ for each subject's probabilities of each ability level. The mean of the uncertainty values was $-1.414(S D 0.179)$, the maximum was -1.602 , and the minimum was -0.750 . 
probability distribution was adapted to the probability distribution of certain information, the latter to that of uncertain information. It should be noted that the uncertainty values of two information types were varied while the expected values were constant. $^{3}$ The subjects were called again and required to select the most certain and uncertain information among a number of sets of the probability distributions with the expected values near 2 (intermediate ability level). The result affirmed that the above two sets of probability distributions were representative of the lowest uncertainty and of the highest uncertainty respectively.

5. Subjects were then required to choose a total of 100 items to work on by themselves within time limits (10 min), i.e. the experimenter instructed that they were free either to choose items appropriate for their ability level or to choose items from the higher item level to get a high score. And then subjects rated the importance of IS in choosing items on 7-point scales ranging from extremely important (3) to extremely unimportant $(-3)$.

6. Next, subjects were told to assume that they now would have a fictitious partner of the same sex and the same age, and perform the same task independently of him or her, and the scores of subjects and partners would be summed up and compared with other groups. The subjects were randomly given either certain or uncertain IGM whose uncertainty was determined in the pilot study. Then, subjects were again required to choose a total of 100 items to work on together but independently of the fictitious partner with-

3 The expected values of the ability assessment were computed from the subject's probability ratings on the scales by assigning the value 1, 2 and 3 to the lower, intermediate and higher ability level, respectively, and by summing up these values each weighted by its subjective probabilities. The expected values of certain and uncertain information were both 2.200 . in $10 \mathrm{~min}$.

7. Subjects rated importance of IGM in task choice on the 7-point scales. Furthermore, to rate relative importance of IGM to IS in task choice the subjects were asked to allocate $100 \%$ between importance of IGM and that of IS.

\section{Results}

The uncertainty values of the prior belief of subject's ability estimation were analyzed by $2 \times 2$ analysis of variance: certain vs. uncertain IS and certain vs. uncertain IGM. Neither main nor interaction effect was significant. The expected values were subjected to 2-way analysis of variance, which also revealed no main or interaction effect. These results showed that the uncertainty values and the expected values of their ability in their prior belief did not vary among the four experimental conditions. The mean of the uncertainty values was -1.341 (SD 0.256) and the mean of the expected values was 1.994 (SD 0.323), which indicated that the expected values in their prior belief belonged to the intermediate level of ability estimation. So, the mean expected values of their prior belief was almost equal to the expected values of IS and IGM.

The practice, the number of transforming the words correctly, was then analyzed by $2 \times 2$ analysis of variance. The main or interaction effect did not reach a significant level, showing that the practice did not vary among the four experimental conditions. The mean of them was 14.911 (SD 4.202).

Table 1 indicates the mean numbers of items selected from each of three item levels when they were given only IS. Uncertain IS made subjects select a greater number of items from both lower and higher item levels than certain IS did $(t=$ $6.311, d f=52, p<.001 ; t=12.673, d f=52$, $p<.001$, respectively), whereas the result was reversed at intermediate item level $(t=21.405, d f=52, p<.001)$. Both certain 
Table 1

Mean and $S D$ of task choice in cach item level given only IS

\begin{tabular}{lcc}
\hline \multirow{2}{*}{ Item level } & \multicolumn{2}{c}{ IS } \\
& Certain & Uncertain \\
Lower & 12.00 & 19.28 \\
& $(10.40)^{\text {in }}$ & $(10.96)$ \\
Intermediate & 64.41 & 38.40 \\
Higher & $(15.74)$ & $(15.21)$ \\
& 24.28 & 42.52 \\
& $(11.56)$ & $(21.85)$
\end{tabular}

a) $S D_{\text {s are in parentheses. }}$

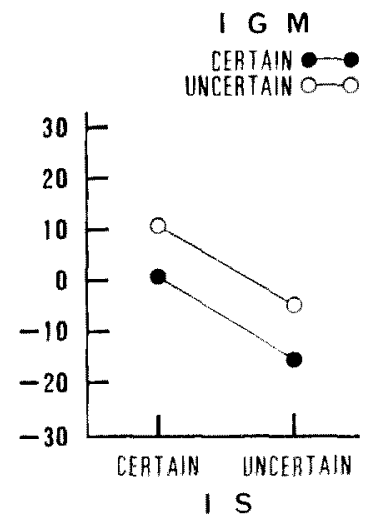

Fig. 1. Mean of choice shift in lower item level as a function of uncertainty of IS and IGM.

and uncertain IS have the expected values matched to intermediate ability level, but by certain IS task choice consisted of a larger number of items from the intermediate item level than by uncertain IS.

The rating of importance of IS in task choice was then analyzed by one-way analysis of variance, including two modes of uncertainty of IS. The rating did not differ significantly between certain (Mean 0.805 ) and uncertain (Mean 1.045) IS. Since the mean was around 1.000 , IS was judged important for task choice regardless of uncertainty.

Now we turn to choice shift, i.e. the subtraction of task choice when given IS only from task choice when IGM is added. Figures 1, 2, and 3 show the mean of

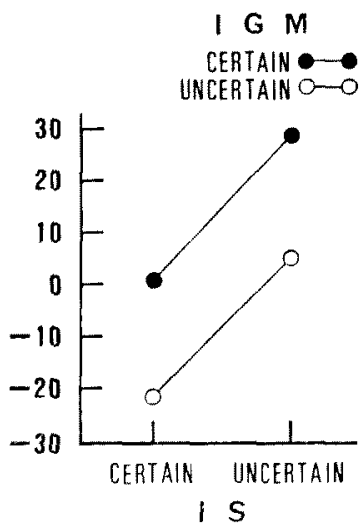

Fig. 2. Mean of choice shift in intermediate item level as a function of uncertainty of IS and IGM.

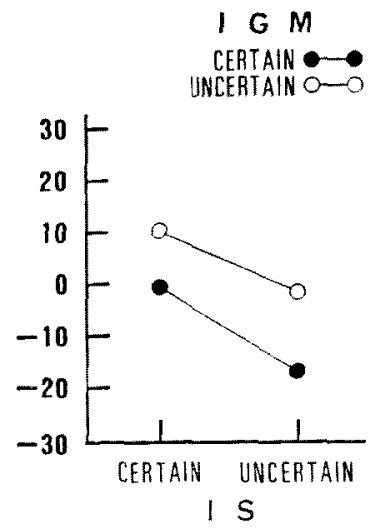

Fig. 3. Mean of choice shift in higher item level as a function of uncertainty of IS and IGM.

choice shift in each item level. The choice shift by arc sine transformation was analyzed by $2 \times 2$ analysis of variance including two modes of uncertainty of IS and two modes of uncertainty of IGM in each item level. All of these three analyses of variance yielded significant main effects for the modes of uncertainty of IS ( $F(1$, $50)=17,667, p<.001$ in lower item level, $F(1,50)=51.302, p<.001$ in intermediate item level, and $F(1,50)=9.087, p<.005$ in higher item level), and the modes of uncertainty of $\operatorname{IGM}(F(1,50)=27.825, p<$ .001 in lower item level, $F(1,50)=36.295$, $p<.001$ in intermediate item level, and 
$F(1,50)=7.589, p<.01$ in higher item level). As clearly shown in Figs. 1, 2, and 3 , the direction of choice shift revealed a similar pattern in lower and higher item levels, whereas the reversed pattern was found in the intermediate item level. In both lower and higher item levels, uncertain IGM added to certain IS caused a positive choice shift, i.e. more items when IGM was added to IS than when only IS was present, and certain IGM added to uncertain is caused a negative choice shift, i.e. fewer items when IGM was added than when IS only. In the intermediate item level uncertainty combinations as above both presented the direction of choice shift opposite to that in both the higher and lower item levels. However, in all three item levels, both certain IGM added to certain IS and uncertain IGM added to uncertain IS, i.e. the conditions under which IS and IGM are both certain or both uncertain, scarcely brought about any positive or negative choice shift.

Importance rating of IGM in task choice to work on independently in a group was then analyzed by $2 \times 2$ analysis of variance, indicating a tendency for an interaction effect between uncertainty of IS and that of IGM $(F(1,50)=3.102, p<$ $.10)$.

Finally, Fig. 4 indicates relative impor-

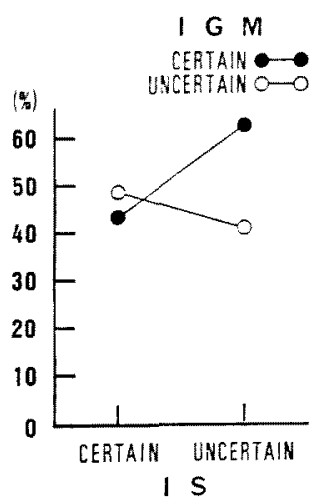

Fig. 4. Mean rating of relative importance of IGM to IS in task choice as a function of uncertainty of IS and IGM. tance of IGM to IS in task choice. This rating of relative importance by arc sine transformation was subjected to $2 \times 2$ analysis of variance, revealing a significant interaction effect between uncertainty of IS and that of $\operatorname{IGM}(F(1,50)=8.107, p<.01)$. When certain IS was given, the ratings of relative importance of certain and uncertain IGM to certain IS did not differ significantly. The mean ratings in these two experimental conditions were both between $40 \%$ and $50 \%$. However, when uncertain IS was given, subjects made more certain IGM than uncertain IGM $(p<.01)$.

\section{Discussion}

Group member's ability estimations including both one's own and other group member's ability estimation were revealed to function as a cue information which determines task choice for group performance. Moreover, uncertainty of these cue information was clearly shown to influence task choice and choice shift. When IS and IGM were heterogeneous in uncertainty, i.e. either when IS was certain and IGM was uncertain or when IS was uncertain and IGM was certain, choice shift took place. And importance of cue information was found to vary depending on uncertainty of cue information. When certain IS was given, subjects rated IS and IGM equally important regardless of uncertainty of IGM. However, choice shift occurred with uncertain IGM but not with certain IGM. In turn, when subjects were given uncertain IS, choice shift took place depending on uncertainty of IGM. But they rated certain IGM more important than uncertain IGM. In other words, choice shift was produced when uncertainty of IS and IGM were in conflict, and the direction of choice shift was in accordance with the probability distribution of IGM. When IS was uncertain and IGM was certain, IGM was rated more important than IS. This indicates 
that IGM might function more signifcantly as a cue than IS. On the other hand, under the condition of certain IS and uncertain IGM, the rated importance did not differ between IS and IGM. It is not clear why choice shift took place in the direction of fitting in the probability distribution of IGM though IS and IGM were rated equal in importance. The results of the present study supported the notions that uncertainty of group member's ability estimations affect the way of their utilization which might finally influence task choice for group performance. Further research is required to determine the relationship between the utilization of group member's ability estimations and task choice for group performance.

\section{References}

Anderson, N. H., \& Graeser, C. C. 1976 An information integration analysis of attitude change in group discussion. Journal of Personality and $S_{0}$ cial Psychology, 34, 210-222.

Atkinson, J. W. 1957 Motivational determinants of risk-taking behaviour. Psychological Review, 64, 359-372.

Burnstein, E., \& Vinokur, A. 1975 What a person thinks upon learning he has chosen different- ly from others: Nice evidence for the persuasive-arguments explanation of choice shifts. Journal of Experimental Social Psychology, 11, 412-426.

Goethals, G., \& Zanna, M. 1979 The role of social comparison in choice shifts. Journal of Personality and Social Psychology, 37, 1469-1476.

Kovacs, Z, 1982 Group decision under uncertainty: Group structure and the shift phenomenon. In H. Brandstatter, J. Davis \& G. StockerKreichgauer (Eds.), Group decision making. London: Academic Press. Pp. 201-214.

Raynor, J. 1982 Future orientation, self-evaluation, and achievement motivation: Use of an expectancy $x$ value theory of personality functioning and change. In N. Feather (Ed.), $E_{x}$ pectations and actions. Hillsdale, N. J.: Lawrence Erlbaum Associates. Pp. 97-124.

Tanaka, H. 1970 The effect of solution word difficulty variables on anagram solution time: Letter order and task word. Japanese Journal of Psychology, 41, 195-204. (In Japanese)

Trope, Y., \& Brickman, P. 1975 Difficulty and diagnosticity as determinants of choice among tasks. Journal of Personality and Social Psychology, 31, 918-925.

Trope, Y., \& Ben-Yair, E. 1982 Task constraction and persistence as means for self-assessment of abilities. Journal of Personality and Social Psychology, 42, 637-645.

(Received Sept. 18, 1986; accepted May 9, 1987) 